\title{
RESEARCH ON EVALUATION OF THE SPALLING DISEASE OF NICHE FOR BUDDHA IN GROTTOES
}

\author{
Y. Miao ${ }^{1 \cdot 2}$, M. Hou ${ }^{1,2 *} *$, P.Wei ${ }^{1.2}$ \\ ${ }^{1}$ School of Geomatics and Urban Spatial Informatics, Beijing University of Civil Engineering and Architecture, No.15 Yongyuan \\ Road, Daxing District, Beijing; 102616, China \\ ${ }^{2}$ Beijing Key Laboratory for Architectural Heritage Fine Reconstruction and HealthMonitoring, No.15 Yongyuan Road, Daxing \\ District, Beijing; 102616, China
}

KEY WORDS: Grottoes protection; Point cloud classification; Random forest; Spalling disease; Quantitative evaluation

\begin{abstract}
:
The niche for Buddha is an important part of the cultural relics in the grottoes, but it has caused serious spalling disease due to human activities and natural disasters. This paper presents a new quantitative evaluation method for the spalling disease of niche for Buddha. First, the random forest-based method was used to identify the niche for Buddha . Then, the spatial clustering of the head of the niche for Buddha was performed by DBSCAN, and the reference surface was determined by the RANSAC algorithm. Based on this, the spallation depth of the grotto surface was extracted and the relevant index elements were established. The experiment of point cloud data in Cave 18 of Yungang Grottoes proves the rationality of this method, which is of great significance to the protection and virtual restoration of cultural relics in grottoes.
\end{abstract}

\section{INTRODUCTION}

As cultural heritage, grottoes have important historical, artistic, cultural and emotional values. However, the grottoes are immovable cultural relics, and with the passage of time, serious weathering diseases have occurred. As the smallest statue unit in the grottoes, the niche for Buddha has the most serious disease of weathering and spalling, so it is in urgent need of an effective protection method. Scientific evaluation of the degree of spalling of niche for Buddha is an important prerequisite for the protection of grottoes and an important indicator to reflect their preservation status.

The traditional spalling depth measurement mainly relies on the manual local measurement method. For example, Wells (Wells, T. et al.,2008) used electronic calipers to measure spalling on the surface of ancient sandstone tombstones. Zhang (Zhang Z.J. et al.,2010a) measured the depth of gouge marks near and inside the cave of Longyou Grottoes with the method of plasticine pressing. Mottershead (Mottershead, D. N., 2000) used a simple probe or steel ruler to make a quantitative observation of the depth of surface depressions at selected representative locations to determine moderate spalling of the rock. In the measurement of the spall degree of the wall sandstone of the ancient city of Pingyao, Shanxi, Zhang (Zhang Z.J. et al.,2010b) inserts the measuring rod of a dial gauge into a small hole, and the measuring head contacts the surface of the cultural relic to be measured. At the same time, the bottom bracket of the dial head contacts the glass plate. At this time, the reading is the distance from the reference surface to the cultural relic surface, and the accuracy of this method is better than the steel needle measurement. However, the traditional methods mentioned above have some problems such as low efficiency and the evaluation results are susceptible to subjective factors, and can't fully reflect the spalling situation of objects.

This paper presents a new quantitative evaluation method for the disease of spalling of niche for Buddha. First, the random forestbased method was used to identify niches for Buddha, and then the method combining DBSCAN and prior knowledge was used to monomer the point cloud of niche for Buddha. Then, the reference surface was determined by using the RANSAC algorithm. Based on this, the spallation depth on the grotto surface was extracted and relevant index elements were established. Through the experiment on the point cloud data of Cave 18 of Yungang Grottoes, the experimental results match with the present situation, which proves the rationality of the method. It can provide scientific basis for the protection and virtual restoration of the grottoes..

\section{RELATED WORK}

3D laser scanning technology has the advantages of high efficiency, high precision, automation and non-contact, etc. Since the 1990s, it has been used in the acquisition of 3D information of grotto cultural relics, which cannot be achieved by traditional technology. In 1999, the Italian cultural relics protection department in collaboration with Stanford university and the university of Washington has launched "Michelangelo digital project" (Levoy, Marc, et al. 2000), using 3D laser scanning technology to 10 including "David" masters of the Italian Renaissance, the digitization, the works of Michelangelo created a 3D laser scanning technology for the protection of cultural relics. Using 3D laser scanning technology, J. H. Park (Park, J. H., et al. 2015) made a complete 3D digital archive of the Seokguram Grottoes in South Korea, a world cultural heritage, and then optimized the scanned data to produce 3D models with high geometric accuracy and visual quality. The completed grottoes were then exhibited in 3D. The research of 3D laser scanning technology started relatively late in China, but in the last ten years, domestic scholars also began to apply $3 \mathrm{D}$ laser scanning technology to the protection of stone cultural relics. The relevant teams of Dunhuang Grottoes (Chen and Fan, 2016.), Yungang Grottoes ( $\mathrm{Lu}$ and Ning, 2016) and Longmen Grottoes (Li,2019)s successively started the point cloud information retrieval and mapping work of the grottoes statues, and made a comprehensive digital archiving record of the overall preservation status of the grottoes.

Three-dimensional laser technology has gradually been used in the study of spalling of stone relics. For example, Andre (Andre et al,2008) used 3D laser scanning and photogrammetry technology to evaluate the degree of rock damage and spalling in the eastern section of the first layer of the 1,000-year-old Takeo Temple. From the point cloud data, Fais (Fais et al,2015).

* Corresponding author : M.Hou, e-mail :houmiaole@bucea.edu.cn 
calculated the geometric anomalies of the surface of the rock materials of the historic Palazzo di CittA in Italy. Based on the research of sandstone spalling depth at home and abroad, Zhang Zhongjian proposed to use TLS equipment to quantitatively calculate the spalling depth of different areas of the object of study, so as to provide theoretical support for the subsequent weathering prevention work in Yungang Grottoes. However, although the current methods have introduced modern surveying and mapping technology, they are still dominated by manual or human-computer interaction. The evaluation method for such a large number of objects as niches for Buddha still lacks convenience in practical operation.

\section{METHODOLOGY}

In order to evaluate the status quo of niches for Buddha scientifically, it is an important prerequisite to identify the objects of niches for Buddha from the wall of grottoes. The reference surface is constructed as the reference plane to calculate the depth of niches for Buddha spalling, and the design index is used as the judgment basis to evaluate the spalling of niches for Buddha.

\subsection{Point cloud classification based on random forest}

In practical work, it is an indispensable step to identify and extract the point clouds of niche for Buddha in order to quantitatively evaluate the degree of spalling of niche for Buddha. But there are so many niches that it is difficult to extract objects from the huge cave scene by hand. Random forest is a popular machine learning method at present. Compared with the current common algorithms, random forest algorithm can better deal with high-dimensional massive data sets. Moreover, based on the double-random idea of selecting samples and features, random forest can effectively avoid the overfitting problem commonly faced by common classifiers and has better generalization performance and robustness.

A large number of features are needed as explanatory variables to ensure the reliability of the decision when the random forest method is used for point cloud identification. In order to improve the classification accuracy of high point cloud, multi-scale feature sets are first constructed as the classification basis. Characteristic parameters of this institute with include four categories: space characteristics (including: $\mathrm{X}, \mathrm{Y}$, and $\mathrm{Z}$ coordinates and Roughness), surface characteristics (including Mean curvature, Gaussian curvature and Normal vector change rate), the density of relevant features (including: Number of neighbors, Volume density, Surface density), the spatial distribution characteristics (As shown in table 1)(including: Planarity, Linearity, Sphericity, Anisotropy).

The respective derived eigenvalues $\lambda \_i$ with $i \in\{1,2,3\}$ within a certain neighborhood can be used to explore and quantize local $3 \mathrm{D}$ shape. The eigenvalues are firstly normalized into $\eta \_i$ with $\mathrm{i}$ $\in\{1,2,3\}$.

\begin{tabular}{|c|c|c|}
\hline Planarity & $P_{\eta}=\frac{\left(\eta_{2}-\eta_{3}\right)}{\eta_{1}}$ & \multirow{4}{*}{$\eta_{i}=\frac{\lambda_{i}}{\sum_{i=1}^{3} \lambda_{i}}$} \\
\hline Linearity & $L_{\eta}=\frac{\left(\eta_{1}-\eta_{2}\right)}{\eta_{1}}$ & \\
\hline Sphericity, & $S_{\eta}=\frac{\eta_{3}}{\eta_{1}}$ & \\
\hline Anisotropy & $A_{\eta}=\frac{\left(\eta_{1}-\eta_{3}\right)}{\eta_{1}}$ & \\
\hline
\end{tabular}

Table 1. Formula of spatial distribution feature calculation When the experimental sample set contains a large number of features, it may contain unreasonable redundant features. These redundant features will not only slow down the recognition speed and increase the hardware cost, but more importantly, will directly affect the construction of the decision tree and eventually reduce the accuracy of classification. Therefore, it is a key step to select sensitive and useful features from massive data samples to participate in classification. Variable Importance Measure (VIM) is used as the method of feature importance evaluation in point cloud data. After the feature importance assessment of the point cloud is completed, the importance of the point cloud is sorted, and the feature with the lowest importance is iteratively deleted from the current feature set by the sequence-item elimination algorithm to form the best feature collection. Finally, random forest is used to complete point cloud classification. In addition, in order to ensure the authenticity and reliability of the final training model, the training strategy of Kfold cross-validation method was adopted in this paper for verification.

\subsection{Monomer of niche for Buddha and determination of reference surface}

In order to automate the analysis of the depth of rock spalling for individual niche for Buddha, it is necessary to make each niche a separate object. Therefore, when the random forest is used to complete the point cloud classification, all niches for Buddha are divided into the same label, and all objects are clustered to complete the monomer process. The size of the head and edge of the shrine is relatively fixed, which has obvious semantic characteristics. This prior knowledge can be incorporated into DBSCAN algorithm as a semantic condition (Rudolf and Kristian. 2020). Firstly, this paper specifies the number of point clouds in the cluster and eliminates most of the noise. Then, it limits the size of the cluster and completes the final clustering process of the Niche for Buddha and Buddha head.

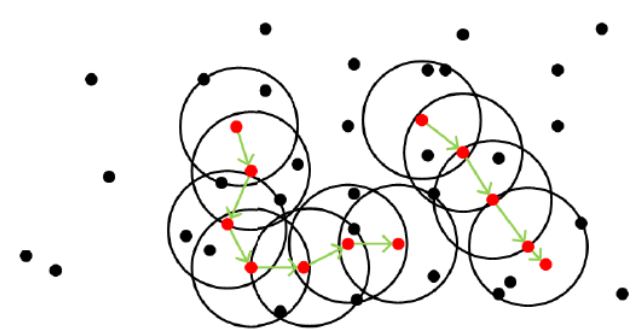

Figure 1. Schematic diagram of DBSCAN segmentation algorithm

The establishment of reliable reference plane is the premise of determining spalling depth and depth direction. This paper adopts the method of plane fitting from point cloud data to establish the reference surface. The methods of plane fitting from point cloud data include Least Squares Fit, Eigenvalue fitting and Random Sample Consensus (RANSAC) algorithm. However, considering that the point cloud in the actual scene is disturbed by noise and other objects, the plane fitting algorithm selected should ensure strong anti-noise performance. For nonplane objects such as niche for Buddha walls, the least square fitting method and eigenvalue fitting method can not effectively deal with gross errors and outliers in the objects when estimating the plane. Therefore, this paper uses RANSAC algorithm to estimate the plane of the point cloud in the target area.

Random sampling consistency algorithm (RANSAC) is an effective robust estimation method. It was first proposed by Fischler and Bolles in 1981, and has been widely used in the fields of machine vision and model parameter estimation since 
then. It is the most robust parameter estimation method at present. The estimation data of RANSAC algorithm contains correct points and noise points, in which the correct points are inliers and the invalid points are outliers. Different from the least square algorithm, which uses all the points for fitting, RANSAC only estimates according to the local points, and the outside points as noise do not participate in the fitting.

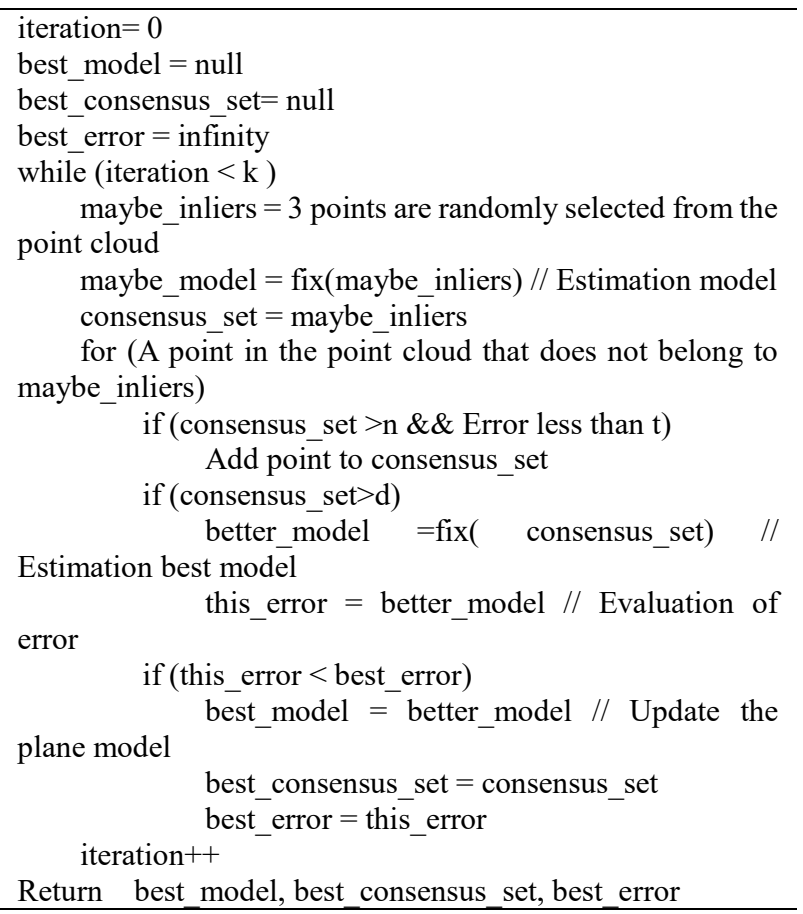

\subsection{Evaluation method of spalling disease in grottoes}

The rock spalling index of niches for Buddha is an important parameter to evaluate the degree of spalling, and the scientific quantitative index is helpful to formulate effective protection measures, which is of great significance for the protection and restoration of grottoes. In engineering, single point extraction of spalling depth is generally carried out by manual method, so it is impossible to make a comprehensive quantitative analysis of the severity of spalling disease. The severity of spalling disease is also measured visually by subjective experience. However, these methods lack scientific basis, are inefficient, and rely too much on expert knowledge and experience. In order to analyze the severity of spalling, we need to design a relevant evaluation index that can comprehensively reflect the situation of spalling. Traditionally, people often choose a measurement point on cultural relics to compare with the lowest point of spalling in grottoes, so as to reflect the degree of spalling. However, the spalling depth of a single location has some limitations. To truly reflect the extent of the target's flaking, the data must reflect the overall flaking. Taking the spalling disease of cultural relics in grottoes as the research object, this paper puts forward a disease index which can reflect the maximum spalling depth and the average spalling depth in one, so as to explore and improve the present situation of judging the degree of spalling of rocks according to experience.

The maximum spalling depth difference $D r$ reflects the dispersion degree of spalling depth of each niche for Buddha, which is the extreme difference of spalling depth. Its mathematical representation is shown in Formula 1.

$$
\text { Dr }=\text { Dmax }- \text { Dmin, }
$$

In the formula, Dmax represents the maximum depth value in the currently measured point cloud of niche for Buddha relative to the datum surface, and Dmin represents the minimum depth value in the currently measured niche for Buddha point cloud relative to the datum surface.

Due to the plane model fitted by RANSAC in this paper, it can be expressed as Formula 2.

$$
\mathrm{ax}+\mathrm{by}+\mathrm{cz}=\mathrm{d} \text {, }
$$

Therefore, the maximum spalling depth difference can be expressed as Formula 3.

The average spalling depth difference Dmean can reflect the overall situation of spalling of each niche, and its mathematical expression is shown in Formula 4.

$$
D_{\text {mean }}=\frac{\sum_{i=1}^{n}\left(D_{\max }-D_{i}\right)}{n}
$$

Combined with the normal depth vector parameters, the final calculation method of average spalling depth difference is shown in Formula 5.

$$
\begin{gathered}
D_{r}=a\left(x_{\text {max }}-x_{\text {min }}\right)+b\left(y_{\text {max }}-y_{\text {min }}\right)+c\left(z_{\text {max }}-z_{\text {min }}\right), \\
D_{\text {mean }}=\frac{\sum_{i=1}^{n}\left[a\left(x_{\text {max }}-x_{i}\right)+b\left(y_{\text {max }}-y_{i}\right)+c\left(z_{\text {max }}-z_{i}\right)\right]}{n},
\end{gathered}
$$

Since the maximum and minimum values of the spalling index are unknown, and the problem of outlier data points may exist, this paper uses the Z-score standardization method to propose a calculation model of the spalling index suitable for spalling disease, which can be expressed as Formula 6.

$$
D_{\text {score }}=\frac{D_{r}-\mu_{D_{r}}}{\sigma_{D_{r}}}+\frac{D_{\text {mean }}-\mu_{D_{\text {mean }}}}{\sigma_{D_{\text {mean }}}},
$$

Where:

$\mu$ is shown in Formula 7 and $\sigma$ is shown in Formula 8

$$
\mu_{x}=\frac{1}{n} \sum_{i=1}^{n} x_{i},
$$

$$
\sigma_{x}=\sqrt{\frac{1}{n-1} \sum_{i=1}^{n}\left(x_{i}-\mu_{x}\right)^{2}},
$$

\section{RESULTS AND DISCUSSION}

\subsection{Description of the Datasets}

Yungang Grottoes, located in Datong City, Shanxi Province, China, was first built in the 5th century. There are more than 40 caves, more than 1,200 niche for Buddhas and more than 50,000 stone statues. It is one of the largest grottoes in the world and represents the highest level of sculpture in China in the fifth 
century. It has been listed as a World Cultural Heritage Site. Among them, Cave 18 of Yungang Grottoes, one of the earliest "five caves by Tan Yao", is the one with the most complete structure and exquisite carving, as shown in the picture. The eighteen grottoes are magnificent, and the grottoes are elliptical in plane and domed in roof. The grottoes are horseshoe-shaped and gradually shrink from bottom to top. The overall height of the grottoes is about 17 meters, the width of the grottoes is about 17.5 meters, and the depth of the grottoes is about 8 meters. The main Buddha statue on the north wall is 15.5 meters high. There are statues of Buddha and Bodhisattva statues on the east and west sides, and a large number of niches for Buddha on the south wall.

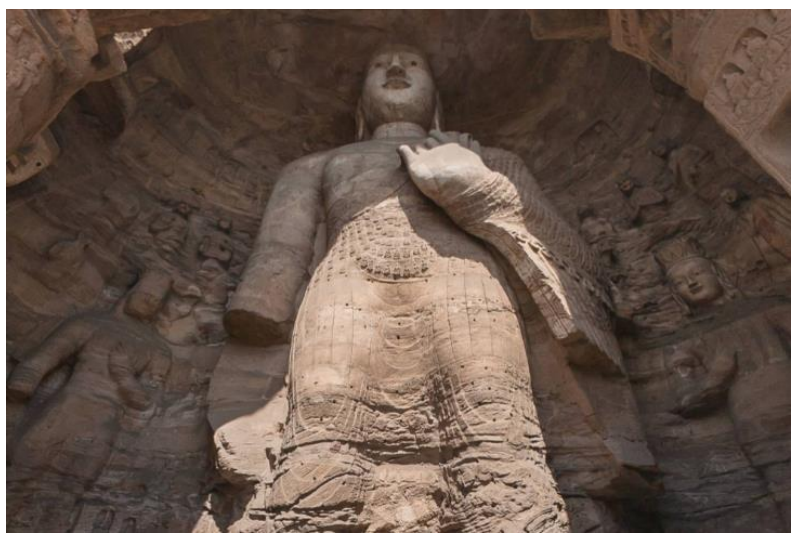

Figure 2. Yungang Grottoes Cave 18

In this paper, point clouds on the east of the south wall of Cave 18 of Yungang Grottoes are selected as the research object. After point cloud denoising and resampling, the final number of point clouds is 381,502 , as shown in the figure 3 .

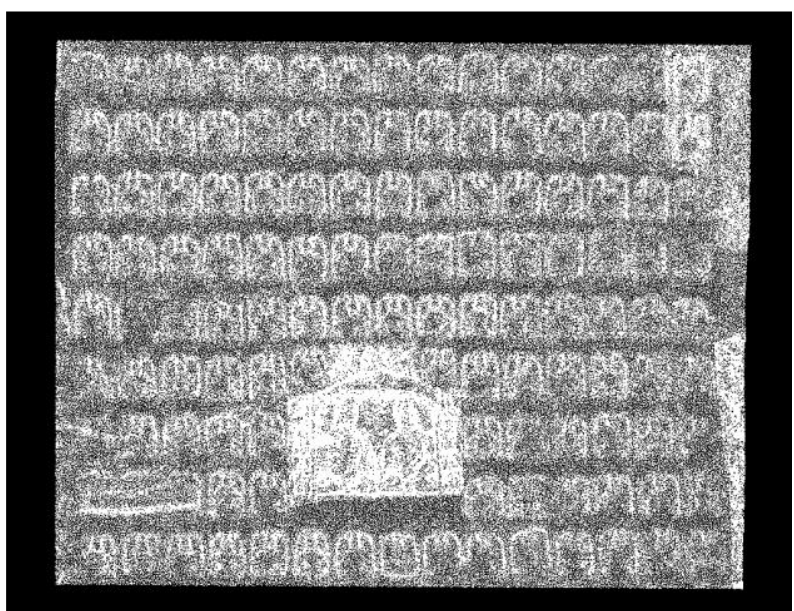

Figure 3. Point cloud data on the east side of the south wall of the Cave 18

\subsection{Results of random forest classification}

This paper uses the variable importance scoring method to rank the importance of features, and the ranking results are shown in the figure below. It can be seen from the figure that the mean value of the inner product of the normal vector accounts for a maximum of 0.16 in the classification of point cloud, which is related to the great difference of normal distribution on the surface of grottoes. The second is that the $Z$-value is about 0.15 , mainly because the $\mathrm{Z}$-value can reflect the change of depth. For features with low importance, the first consideration should be taken in feature selection.

According to the ranking result of the overall feature importance and the construction method of the point cloud highly sensitive feature set, the features with low Vim value were deleted from high to low in order, and the new features were reconstructed, and the accuracy of point cloud recognition was recalculated by using the feature set. In this paper, the minimum value of the feature set is set to 3 . After iterative testing, the final features selected in this paper are: the mean value of the Normal change rate, the Mean curvature, the Sphericity index, the Surface density, the Gaussian curvature and the Number of neighbors. The final feature importance measure is shown in the figure.

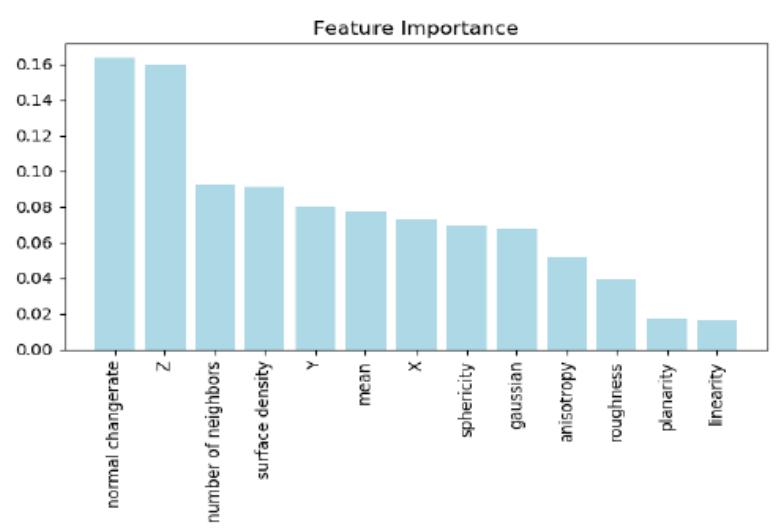

Figure 4. Feature importance statistics before feature selection

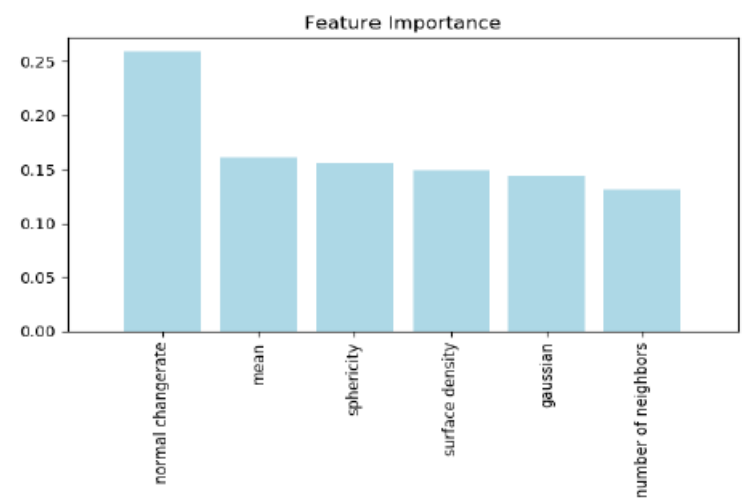

Figure 5. Feature importance statistics after feature selection

In the process of the classification of the random forest, in addition to optimize the feature set, but also to the random forest model parameters are adjusted in order to achieve the best classification purpose. Network style parameter is a kind of super parameter optimization algorithm, which can automatically obtain more reasonable parameters in most cases, rather than relying on manual experience. Finally, the number of decision trees Ntree is set as 100, and the feature number of splitting is set as 4 . The results before and after feature selection are shown in the figure. 


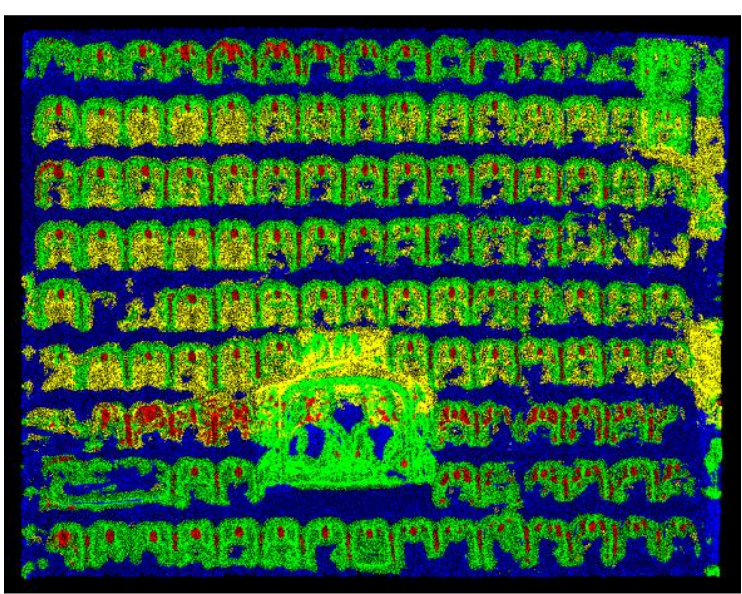

Figure 6. Classification result of point cloud before grotto selection

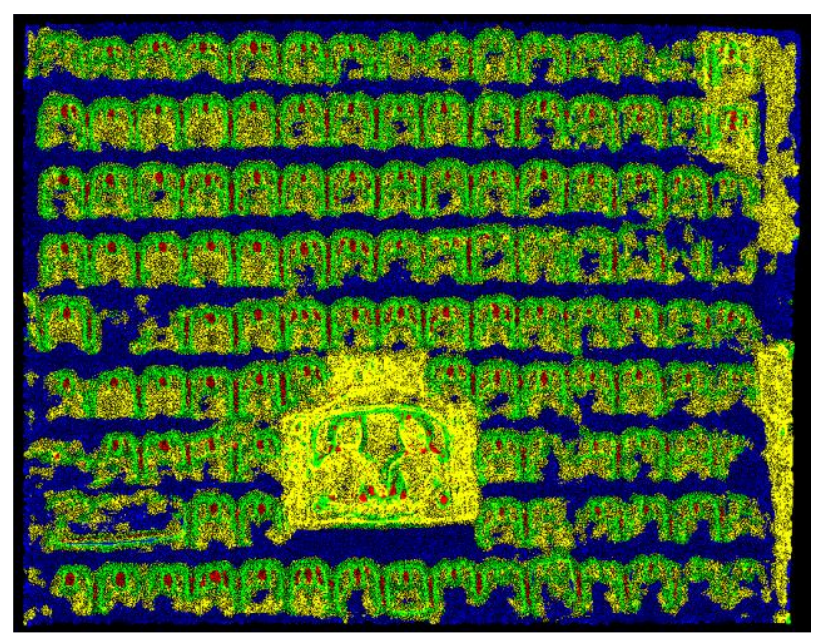

Figure 7. Classification result of grotto point cloud after feature selection

In order to objectively evaluate the classification effect of this study, the classification results were evaluated by quantitative analysis indicators. The overall accuracy (OA), Kappa coefficient and time consuming before and after feature selection are shown in Table 1.

$$
\begin{gathered}
\mathrm{OA}=\frac{\sum_{i=1}^{n} n_{i i}}{N} \times 100 \% \\
\text { Карра }=\frac{\sum_{i=1}^{n} n_{i i}-\sum_{i=1}^{n} n_{i}-n_{i}^{\prime}}{N \times N-\sum_{i=1}^{n} n_{i}-n_{i}^{\prime}}
\end{gathered}
$$

Where:

$\mathrm{N}=$ the number of point clouds in the sample

$n_{i i}=$ the number of point clouds correctly predicted by

Class I labels in the mixed matrix

$n_{i}=$ the total number of Class I point clouds in the test set

$n_{i}^{\prime}=$ the number of point clouds of Class I tags in the prediction model.

\begin{tabular}{cccc}
\hline & OA & Kappa & Time (s) \\
\hline Before & 0.697 & 0.664 & 163 \\
After & 0.788 & 0.771 & 121 \\
\hline
\end{tabular}

Table 2 The overall accuracy and Kappa coefficient of the point cloud classification

It can be seen from the quantitative analysis results of the classification effect that the feature selection algorithm based on the importance rating of variables can significantly improve the effect and efficiency of the classification after feature selection, which also verifies the effectiveness of the feature selection algorithm in this paper.

\subsection{Results of monomer of niche for Buddha}

In the actual operation, this paper uses the PyPCL module in python to cluster DBSCAN point clouds, and sets the minimum number of point clouds $\mathrm{MP}=20$ and neighborhood radius Ep $=35 \mathrm{~mm}$. According to the analysis results of the head of the Buddha, the semantic rules are set as follows: the total number of point clouds in a single cluster is $40-80$, and the length in the $\mathrm{X}$ direction is within the range of $28-48 \mathrm{~mm}$, and the length in the $\mathrm{Z}$ direction is within the range of $40-60 \mathrm{~mm}$. After DBSCAN clustering for the first time, the effect is shown in Figure 5-7. It can be seen that most of the noise has been eliminated after clustering, and the point clouds of Buddha head are basically clustered into different clusters, but there are still a few nontarget point clouds that are wrongly clustered. The effect after semantic filtering is shown in Figure 5-8. Through semantic rules, non-target points have been basically filtered out, and most of the heads of Buddha are correctly divided into different clusters.

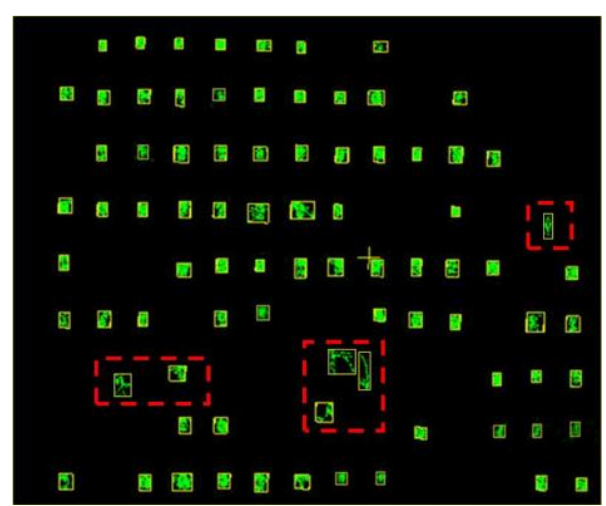

Figure 8. The original classification result of the head of the niche for Buddha

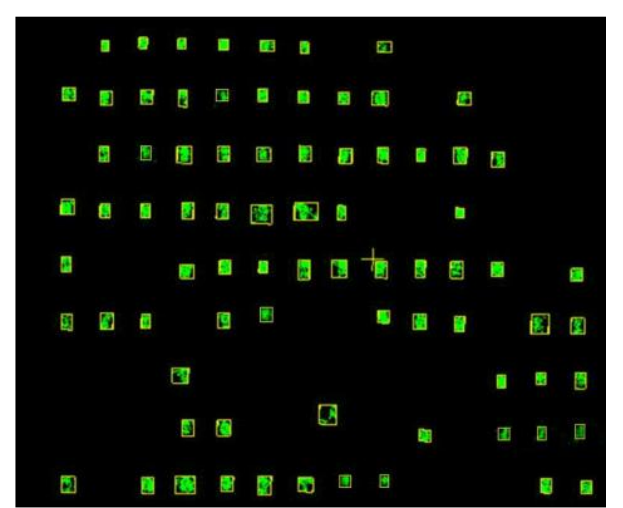

Figure 9. Clustering effect after semantic filtering 


\subsection{Results of monomer of niche for Buddha}

The reference surface of the data is calculated by RANSAC algorithm, which is the premise of calculating the spalling depth. After determining the reference surface, this paper first numbered them according to the distribution law according to the point cloud data of the heads of 78 niches for Buddha after clustering, as shown in the figure 10, Then the maximum spalling depth difference $D r$ and average spalling degree difference of each sample were extracted for statistical analysis and experimental verification. The maximum spalling depth difference is used for statistical analysis and experimental verification, the spalling disease index was calculated, and it was returned to the range of $0-100$.

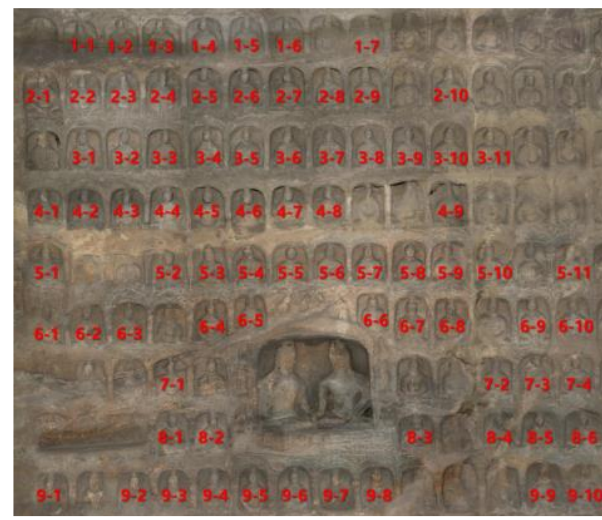

Figure 10. Schematic diagram of niche for Buddha numbering

\section{Results}

\begin{tabular}{|c|c|c|c|c|c|c|}
\hline Picture & & & & & & \\
\hline Number & $4-9$ & $9-10$ & $1-7$ & $1-6$ & $9-3$ & $2-4$ \\
\hline Spalling index & -5.18258 & -4.41317 & -2.68498 & -1.70764 & -0.51755 & -0.00193 \\
\hline Regression & 0.0 & 6.7 & 21.8 & 30.3 & 40.7 & 45.2 \\
\hline \multicolumn{7}{|l|}{ Picture } \\
\hline Number & $3-3$ & $1-3$ & $9-1$ & $5-8$ & $5-7$ & $6-8$ \\
\hline Spalling index & 0.491254 & 1.123396 & 1.817807 & 2.883945 & 4.717748 & 6.281397 \\
\hline Regression & 49.5 & 55.0 & 61.1 & 70.4 & 86.4 & 100.0 \\
\hline
\end{tabular}

Table 3. Comparison of quantitative evaluation results

To verify the rationality of the model proposed in this paper, it is necessary to compare the final calculation results with the current photos of the real preservation status of Yungang Grottoes. In this paper, representative samples are selected and shown in Table 3 in the order of spalling index from small to large. As you can see, the number is. It can be seen that the head of the niche for Buddha No. 49 has the most severe spalling degree and the lowest spalling index. With the increase of the spalling index value, the damage degree of the niche for Buddha gradually decreases. Since the present situation and the spalling index show the same changing trend, in summary, the establishment of the model is reasonable and feasible.

Through the final calculation results of the spalling index and the field survey on the south wall of Yungang Eighteen Grottoes, it was found that the spalling index near the entrance of the cave was generally low, and the spalling disease was serious. This result may be related to the influence of light, rain, wind erosion and other environmental factors on the niches for Buddha near the cave entrance. At the same time, the spalling index of
Buddhist altars distributed in lower altitudes such as the eighth row and ninth row is generally smaller than that of Buddhist altars distributed in higher altitudes such as the first row and second row. Description Overall, compared to the shrines at the low, high shrines save the situation better, affected by spalling of smaller, these differences may be related to long-standing shrines at low humidity, the greater the influence of temperature related. In addition, related research based on the spalling index of niches for Buddha can also provide a theoretical basis for the advancement of Chinese grotto weathering technology and promote the development of related theories.

\section{CONCLUSION}

The spalling of cultural relics in grottoes is becoming more and more serious. Scientific assessment of the status quo of the spalling of niche for Buddha is the premise of restoration and protection. In this paper, a new quantitative assessment method of niche for Buddha spallation disease was proposed. First, the random forest-based method was used to identify the niche for 
Buddha. Then, DBSCAN was used to cluster the head of the niche for Buddha and RANSAC algorithm was used to determine the reference surface. Finally, the spalling degree index was constructed comprehensively. The experiment on the data of Cave 18 of Yungang Grottoes proves that the method and related process proposed in this paper can better reflect the severity and preservation status of grottoes spalling and fill the blank of quantitative evaluation of grottoes spalling degree. In the future research, experiments will be conducted on more data to prove the versatility and scientificity of this method in more grottoes.

\section{ACKNOWLEDGMENTS}

We would first like to thank the anonymous reviewers for their valuable feedback. We appreciate the support of the Young Beijing Scholars Program

\section{REFERENCES}

Andre, M. F. , Etienne, S. , D Mercier, Vautier, F. , \& Voldoire, O. ,2008. Assessment of sandstone deterioration at ta keo temple (angkor): first results and future prospects. Environmental Geology, 56(3-4), 677-688

Cao C.P., Wang H.Q., Wang K., Wang Z., Ma T., 2020. Intelligent Evaluation Method of Grottoes Surface Weathering Based on Multispectral Imaging and Random Forest Algorithm. Acta Optica Sinica, 40(22):2230001.

Chen Z.W., Fan J.S., 2016. Application of Cultural and Technological Convergence in Cultural Heritage Protection. Dunhuang Reserch. (2),100-107.

Fais, S. , Cuccuru, F. , Ligas, P. , Casula, G. , \& Bianchi, M. G. , 2015. Integrated ultrasonic, laser scanning and petrographical characterisation of carbonate building materials on an architectural structure of a historic building. Bulletin of Engineering Geology \& the Environment.

Guinard, S., \& Landrieu, L., 2017. Weakly supervised segmentation-aided classification of urban scenes from 3D lidar point clouds.

Levoy, Marc, et al. 2000.The digital Michelangelo project: 3D scanning of large statues. Proceedings of the 27 th annual conference on Computer graphics and interactive techniques.

Lu J.W., Ning B., 2016. The Exploration and Practice of Digital Cultural Relics in Yungang Grottoes. Research on Heritages and Preservation

Li Y., 2019. Discussion on the Construction of Grotto Cultural Relic Digitization -- Taking Longmen Grottoes as an Example. Cultural Relics Identification and Appreciation, No.163(16), 103-103.

Mottershead, D. N.,2000 . Weathering of coastal defensive structures in southwest England: a 500 year stone durability trial. Earth Surface Processes and Landforms ,25.10.

Park, J. H., Muhammad, T., \& Jae-Hong, A., 2015. The 3D reconstruction and visualization of Seokguram Grotto World Heritage Site. IEEE.

Rudolf Scitovski,Snježana Majstorović \& Kristian Sabo. 2020. A combination of RANSAC and DBSCAN methods for solving the multiple geometrical object detection problem. Journal of Global Optimization,.

Wittmann, B. M., Stirdivant, S. M. , Mitchell, M. W. , Wulff, J. E. , Mcdunn, J. E. , \& Zhen, L. , 2014. Bladder cancer biomarker discovery using global metabolomic profiling of urine. Plos One, 9(12), e115870.

Wells, T. , Hancock, G. , \& Fryer, J. . 2008. Weathering rates of sandstone in a semi-arid environment (hunter valley, australia). Environmental Geology, 54(5), 1047-1057.

Zhang Zhongjian, Zhang Luqing, Li Lihui, Wang Xueliang, Fu Yan, \& Li Fengxia. 2010a. Influence of Micro-environment to Pelitic Siltstone as Surrounding Rock of Longyou Underground Caverns. Journal of Engineering Geology.

Zhang Zhongjian, Yang Zhifa, Bian Binglei, \& Zhang Luqing. $2010 \mathrm{~b}$. Weathering rates of sandstone in lower walls of Ancient City of Pingyao. Chinese Journal of Geotechnical Emgineering. 032(010), 1628-1632. 